\title{
The Computer-Assisted Interpretation of Copolymer Mass Spectra
}

\author{
Paul O. Danis and Francois J. Huby \\ Research Laboratories, Rohm and Haas Company, Spring House, Pennsylvania, U.S.A
}

A computer program called MSCOPOL has been developed to aid in the interpretation of copolymer mass spectra. The program reads the mass spectrum, calculates the most likely monomer masses via correlation or Fourier transform methods, determines possible end group masses based on the monomer masses, and can then search monomer and end group data bases for likely chemical moieties. Refinement of the end group result is possible by calculation of the monomer ratio and degree of polymerization as a function of end group mass. The program is written in Microsoft Visual Basic and runs on an IBM compatible PC. Applications are shown for polystyrene, poly( $N$-vinyl pyrrolidone/vinyl acetate), and poly(ethylene oxide/propylene oxide). (J Am Soc Mass Spectrom 1995, 6, 1112-1118)

W ith the suite of analytical mass spectrometry methods currently available, production of intact ionized molecules from polymeric materials has become routine. Soft ionization techniques such as field desorption [1-3], fast-atom bombardment $[4,5]$, laser desorption $[6,7]$, secondary ion mass spectrometry $[8,9]$, and matrix-assisted laser desorptionionization [10-12] allow one to obtain mass spectra that show the distribution of molecular ions of a synthetic polymer. When the individual molecular ions can be mass resolved from one another, then the spectrum yields information with regard to the monomer and end group masses as well as the degree of polymerization. The advantage of mass spectrometry over other techniques of polymer analysis, such as infrared and nuclear magnetic resonance spectroscopies [13] is that for low molecular weight materials the distribution of mass as well as the monomers and end groups of the polymer are determined in the same measurement. The spectra can be relatively simple as in the case of a homopolymer where a pattern of peaks are separated by the mass of the monomer. Alternatively, when more than one monomer is used in the preparation of the polymer the spectra are more complicated and the compositional information is much more difficult to extract. For a copolymer molecular ion the quantities that must be determined are the mass of the monomers, the number of each monomer, and the mass of the end groups. Because a limited number of monomers and end groups are actually present in most polymers, knowledge of the mass often is sufficient to conclude its composition. This information is of great utility for fundamental understanding of poly-

Address reprint requests to Dr. Paul O. Danis, Research Laboratories, Rohm and Haas Company, 727 Norristown Road, Spring House, PA 19477-0904. merization mechanisms and monomer reactivity, as well to identify the nature and synthetic routes of unknown materials.

To aid the interpretation of these spectra we have developed a program called MSCOPOL to show us the possible compositions of the ions. Although several approaches have been taken for the interpretation of polymer mass spectra [14-16], the method presented here is unique in that no assumptions are made about the mass or number of the monomers and end groups. Similar to the well known mass spectrometry software tools of probability based matching (PBM) and selftraining interpretative and retrieval system (STIRS) [17], MSCOPOL was developed to be applicable to the total unknown. The algorithm we have developed will read the mass spectrum, find the most likely monomer and end group masses, and search a data base for possible monomers and end groups. It is written in Microsoft Visual Basic for DOS and runs on an IBM compatible personal computer.

\section{Experimental}

Measurements were performed on a JEOL HX-110 mass spectrometer with an acceleration potential of $10 \mathrm{kV}$, mass resolution of 1000 at $10 \%$ valley, and detection of positive ions. For field desorption (FD) measurements, approximately $1 \mu \mathrm{L}$ of $\approx 1 \% \mathrm{wt} / \mathrm{v}$ solution of the polymer was deposited on the carbon emitter. The polystyrene was dissolved in toluene. The poly(ethylene oxide/propylene oxide) was dissolved first in water and then extracted with methylene chloride to reduce the amount of sodium in the sample. This helps ensure that ionization by protonation prevails over sodium cation attachment. An $11.5 \mathrm{kV}$ desorption potential was applied with emitter heating $0-25 \mathrm{~mA}$ at 1-2 $\mathrm{mA} / \mathrm{min}$. The instrument was scanned from 100 
to 3000 in $20 \mathrm{~s}$ and the spectra shown are the sum of several scans. For fast-atom bombardment (FAB), polymer was dissolved in acetone and mixed with 3-nitrobenzyl alcohol matrix on the probe. Samples were ionized with a 6-keV Xe atom beam and the instrument was scanned as before. The polystyrene of average molecular weight $580 \mathrm{u}$ was obtained from Polymer Laboratories, Inc. (Amherst, MA) the poly(ethylene oxide/propylene oxide) molecular weight $1100 \mathrm{u}$ was from Polysciences, Inc. (Warrington, PA), and the poly ( $N$-vinyl pyrrolidone/vinyl acetate) 60/40 copolymer of average molecular weight $2400 \mathrm{u}$ was from Scientific Polymer Products, Inc. (Ontario, NY).

The MSCOPOL program was run from the DOS environment on an IBM compatible personal computer with $16 \mathrm{MB}$ of random access memory with a $486 / 66-$ $\mathrm{MHz}$ processor; also it can be run from a Windows environment. The program requires at least $512-\mathrm{kB}$ memory, < 1-MB disk space, and a minimum processor of 8086. Translation and recompilation would allow it to be used on a Macintosh or VAX system.

\section{Mathematical Solution}

Given a copolymer made of two monomers of mass $M_{1}$ and $M_{2}$ the molecular weight of a polymer molecule, $M$, is equal to the sum of the masses of its monomer units and of its end groups, END:

$$
M=E N D+n_{1} \cdot M_{1}+n_{2} \cdot M_{2}
$$

To fully describe each polymer molecule one needs to determine the monomer masses, the end groups mass, and the number of monomer units, $n_{1}$ and $n_{2}$. The degree of polymerization is a common term for $n_{1}$ and $n_{2}$.

The masses of the monomers are determined by translation of the spectrum + or - DELTAM mass units and correlation of the abundances of the translated spectrum with those of the original spectrum. The highest correlation coefficients arise with translations that satisfy the equation

$$
\operatorname{DELTAM}=j_{1} \cdot M_{1}+j_{2} \cdot M_{2}
$$

where $j_{1}$ and $j_{2}$ are integral. Generally the highest correlation coefficients correspond to $j_{i}=-1,0$, or +1 . A monomer database is available to search for monomers of mass DELTAM.

Alternatively, the monomer masses can be identified by using a Fourier transform algorithm. The mass spectrum of a polymer consists of a series of ions regularly spaced over the mass-to-charge ratio axis. A Fourier transform algorithm should, thus, yield some information about this periodicity that is directly related to monomer masses. The theory tells us that the Fourier transform of a Dirac comb of periodicity $M$ is a single Dirac peak delta(1/M) [18]. The algorithm included in this program calculates the fast Fourier transform (FFT) of the spectrum and plots the modulus of each Fourier transform datum point versus the reciprocal of its abscissa.

For the analysis of the end groups in a linear polymer, one can only determine the sum of the masses of both end groups because no fragmentation of the molecule occurs that would allow differentiation of the two ends of the molecule. For nonlinear polymers the case is somewhat more complicated because three or more end groups would be present. The general solution for end groups is determined by rewriting eq 1 as follows:

$$
M-\operatorname{END}=n_{1} \cdot M_{1}+n_{2} \cdot M_{2}
$$

or

$$
M-\mathrm{END}=\mathrm{GCD} \cdot\left(n_{1} Z_{1}+n_{2} \cdot Z_{2}\right)
$$

where

$$
Z_{i}=M_{i} / \mathrm{GCD}
$$

GCD is the greatest common denominator of $M_{1}$ and $M_{2}$. Dividing both sides of eq 4 by the greatest common denominator gives

$$
(M-\mathrm{END}) / \mathrm{GCD}=n_{1} \cdot Z_{1}+n_{2} \cdot Z_{2}
$$

Because $n_{1}, n_{2}, Z_{1}$, and $Z_{2}$ are all positive or null integers ( $M-$ END)/GCD must be a positive or null integer; otherwise said $M-$ END must be a multiple of GCD. Hence, for a given molecular mass, the sum of the masses of the end groups can only take values that satisfy eqs 7 and 8 :

$$
\begin{gathered}
M=Z \cdot G C D+R \\
\mathrm{END}=R+k \cdot \mathrm{GCD}
\end{gathered}
$$

where the $k$ is a positive or null integer and quantity $Z$ is the result of the integral division of $M$ by GCD with $R$ as the remainder. Thus, eq 9 can replace eq 1 :

$$
Z-k=n_{1} \cdot Z_{1}+n_{2} \cdot Z_{2}
$$

Over the entire spectrum, eq 8 can yield several values for $R$, which will be called root end groups. This means that different end groups are present. The isotopic distribution interferes with the end groups as well to generate multiple $R$ values. Masses that yield different $R$ values must be investigated separately. Moreover if $x$ is a possible end group, $x+p_{1} \cdot M_{1}+$ $p_{2} \cdot M_{2}$ is also a possible end group, where $p_{1}$ and $p_{2}$ are integers. The end groups of mass lower than the lowest mass monomer $\left(p_{1}=p_{2}=0\right)$ form a special class of end groups that cannot be further reduced. These solutions are called basic end groups and are the output given by the program and shown in the following text. The actual end group mass can sometimes be 
derived from a basic end group after the addition of one or two monomer unit masses.

For the determination of the monomer composition of a molecule, the lower mass region (generally $<m / z$ 5000 ) is the most useful. A given $M$, END pair or $Z, k$ pair admits solutions of the form of a two-dimensional vector $\left(n_{1}, n_{2}\right)$. A single pair can have many solutions because a vector $\left(n_{1}+j \cdot Z_{2}, n_{2}-j \cdot Z_{1}\right)$ is also a solution. However, if $j \cdot Z_{1}$ is larger than $n_{2}$, the solution will involve negative components and, thus, become unsatisfactory. Also if $Z>Z_{1} \cdot Z_{2}$, there is one and only one solution vector $\left(n_{1}, n_{2}\right)$, and the most valuable information is contained in the masses less than GCD $\cdot Z_{1} \cdot Z_{2}$ or $M_{1} \cdot M_{2} / \mathrm{GCD}$. Thus, beyond this mass, $M_{1} \cdot M_{2} / G C D$, there are multiple solutions for $\left(n_{1}, n_{2}\right)$ which can satisfy the spectrum.

\section{Description of the Computer Program}

The computer algorithm translates the spectrum DELTAM units and determines the correlation as a function of DELTAM to yield possible monomer masses. The mass of the end groups is then solved as the program goes through all the possible combinations of $k, n_{1}$, and $n_{2}$ and estimates what proportion of the total abundance of the spectrum is accounted for by each value of $k$. Tables and graphic output help the interpreter. Sometimes several values of $k$ satisfy $100 \%$ of the abundance and other considerations have to be taken into account to determine the end groups. Sometimes none of the values of $k$ can account for all the abundance due to the presence of several end groups sums.

The copolymer mass spectra program menu has several options:

Read spectrum, which reads the tabulated spectrum from the PC disk.

Monomers, which determines the probability of various monomer masses that fit the spectrum.

End groups, which determines the probability of various end group masses that fit the spectrum based on the monomers determined.

Isotopic pattern, which uses monomer and end group isotopic distributions to refine the results.

Composition, which uses the preceding results to estimate the monomer ratio in the copolymer.

Databases, which contains the mass, name, formula, and processes of commonly used monomers as well as mass, name, formula, and source of end groups. It is currently being updated to over 250 monomers.

Review results, which lets one view tables of results for masses of monomers and end groups.

Chemical consideration can be taken into account to further solve the problem. The possibilities can be greatly reduced by consideration of chemical information, such as logical compositions and possible end groups from known initiators and chain transfer agents. Often a single unambiguous result is not obtained. However, the greatest asset of this approach is that all possibilities are examined in contrast to the manual approach in which the analyst is often satisfied when a fit is found, and further inquiry is halted.

Several effects that can help reduce the problem are adduct formation, reasonable end groups, current end groups, isotopic contributions, and polymerization process parameters such as reactivity ratios $[19,20]$. The ionization process often occurs by formation of an adduct such as a proton or a sodium or potassium ion bound to the molecule. This has to be taken into consideration when the composition of the end groups is investigated. Obviously some of the masses generated by eq 7 cannot be the actual end group. The minimum end group sum for an $\mathrm{MH}^{+}$ion generated by $\mathrm{FD}$ or $\mathrm{FAB}$ is $\mathrm{H}^{+}$mass $=1 \mathrm{u}$, then $\mathrm{H}_{3}^{+}$mass $=3$, then $\mathrm{CH}_{5}^{+}$mass $=17, \mathrm{H}_{3} \mathrm{O}^{+}$mass $=19$, and so forth. Therefore, a basic end group value often must be complemented by a combination of monomer units to yield a chemically rational end group sum. Although there are many catalysts and chain transfer agents available, only a relatively limited set is commonly used. A list of these agents can be derived from the literature, and the program includes a database facility to store and retrieve current polymer end groups and their origin. The isotopic contribution cannot be neglected in the spectral interpretation. If the monomers are significantly different in composition, the isotopic contribution can yield some helpful information. The spectra must be corrected to take into account the isotopic contributions before abundance-based computations are carried out.

Additionally, for a given $M$, END pair, the computation of the unique solution vector $\left(n_{1}, n_{2}\right)$ is possible only for molecular masses lower than GCD $\cdot Z_{1} Z_{2}$ or $\left(M_{1} \cdot M_{2}\right) / G C D$. This means, for instance, that with monomers of ethylene oxide and propylene oxide the maximum molecular mass that will yield unique solutions for $n_{1}$ and $n_{2}$ is $(44 \cdot 58) / 2$ or $1276 \mathrm{u}$. With $N$-vinyl pyrrolidone and vinyl acetate monomers the value is $(111 \cdot 86) / 1$ or $9546 \mathrm{u}$. However, the limit is usually imposed by the resolving power of the mass spectrometer as opposed to the mathematics. High resolution measurements would increase this limit to larger molecular masses because the GCD would be reduced to a fractional value. If the abundances of each peak are known, one can compute the monomer ratio for the entire sample with good precision. It is possible then to derive information on the polymerization process and the monomer relative reactivity. Conversely, after monomers are determined, the end group mass result can be refined by consideration of the distribution of $n_{1}$ and $n_{2}$, because these values should vary in a fashion consistent with polymerization kinetics [20]. One difficulty may occur when mathematical coinci- 


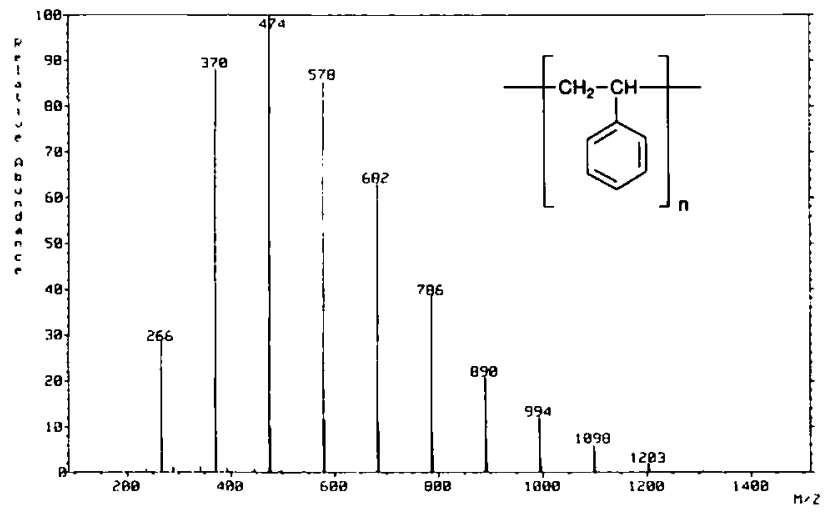

Figure 1. FD mass spectrum of polystyrene $580 \mathrm{u}$.

dences are present such as when the monomers have the same mass or when the mass of one is a multiple of the other. Although this may make the interpretation of the monomer masses more difficult, it will not have an effect on the outcome for the end group.

\section{Results and Discussion}

The method is illustrated with a simple case of the FD mass spectrum of polystyrene with number average molecular weight of $580 \mathrm{u}$, shown in Figure 1. By application of the program for the determination of monomer mass, the result in Figure 2 is obtained where a pattern of peaks is shown $104 \mathrm{u}$ apart. This is, of course, the monomer mass of styrene. The result for translation of the spectrum by zero units is always of unit correlation. Then by entering $104 \mathrm{u}$ as the monomer mass, the end group sum is displayed (Figure 3). The value of $58 \mathrm{u}$ is reasonable as are higher values of 58 plus multiples of $104 \mathrm{u}$. The true value is actually $58 \mathrm{u}$, because this material was prepared in an anionic method from butyl lithium initiator. The isotopic contributions of ${ }^{1,3} \mathrm{C}$ in the molecular ion are evident from the peaks at $\mathrm{m} / \mathrm{z} 59$ and 60 as well as 163

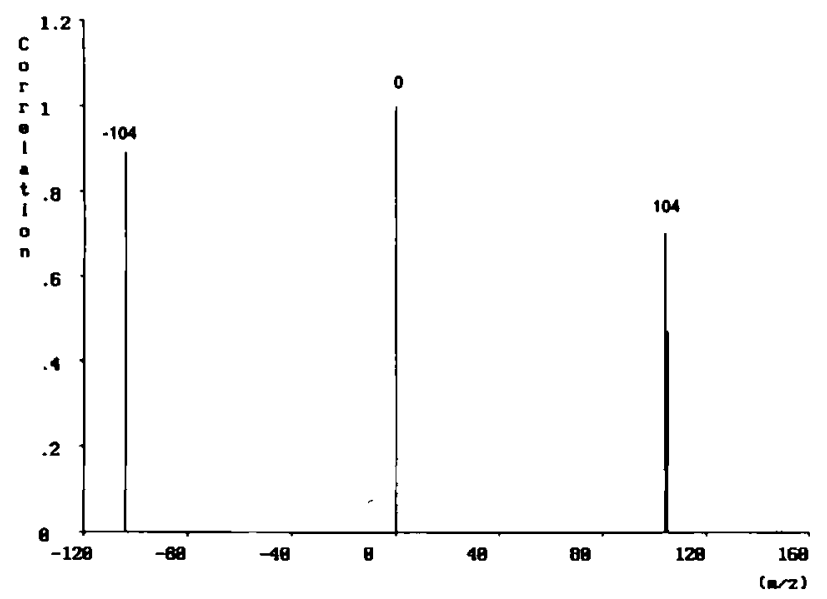

Figure 2. The possible monomer masses for the spectrum in Figure 1.

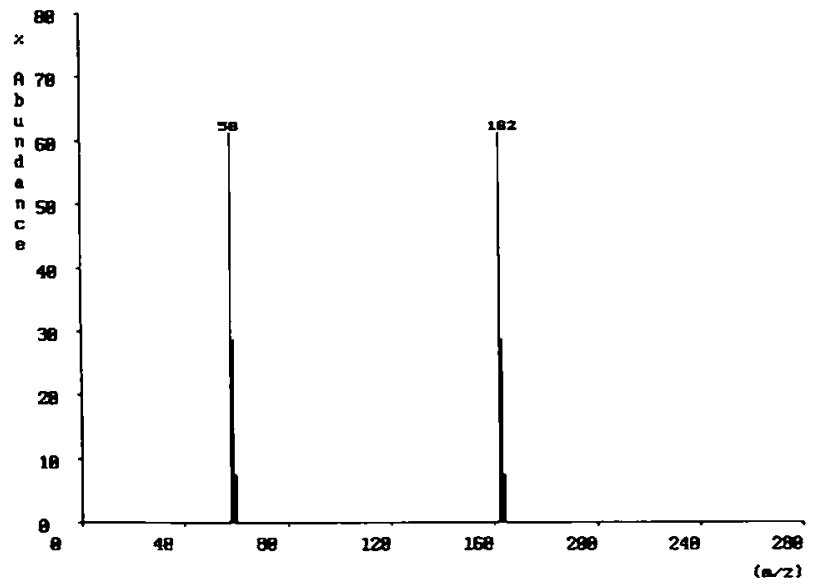

Figure 3. The possible end group masses and the percent of the spectrum in Figure 1 for which they account.

and 164. With an end group of $58 \mathrm{u}$ one also can conclude that the ionization occurred by electron ejection and not attachment of a cation.

Although not present in this spectrum, two effects that could complicate the result are multiple charging and the presence of atoms with high relative abundance of isotopes, such as chlorine or bromine. If doubly charged ions had been produced, as can occur in field desorption, then MSCOPOL would yield monomer and end group masses of half the true values. For the case of isotopic contributions, additional peaks would be observed in the results as before for the ${ }^{13} \mathrm{C}$ isotopes.

The FAB mass spectrum of poly( $N$-vinyl pyrrolidone/vinyl acetate) is shown in Figure 4 for the region of $m /=625-2000$. Ionization in FAB occurs by attachment of a proton or sodium cation to the molecule. The spectrum appears complicated and contains many species of varying composition and mass. The program can rapidly $(<1 \mathrm{~s}$ ) reduce this information to give the result shown in Figure 5 for the most likely monomer masses. The value of $m / z 86$ corresponds to the mass of the vinyl acetate monomer, whereas 111 is from the $N$-vinyl pyrrolidone. The $m /=112$ is also large because

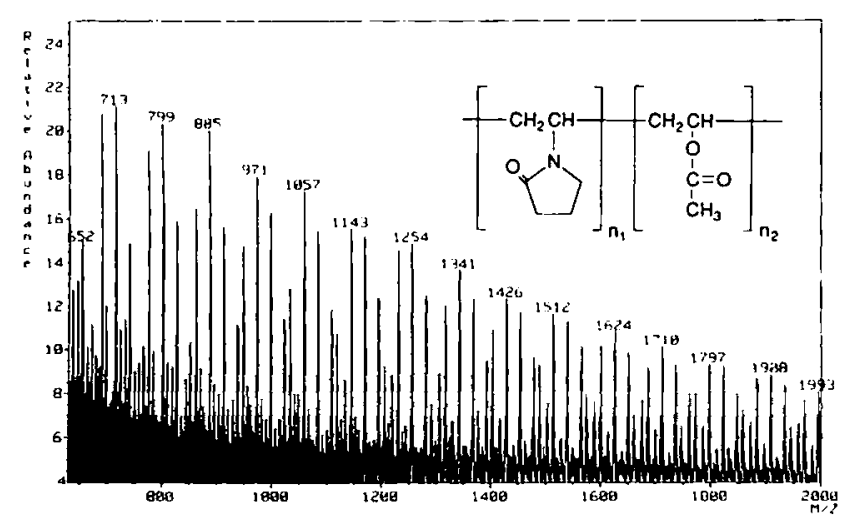

Figure 4. Partial FAB mass spectrum of poly( $N$-vinyl pyrrolidone/vinyl acetate). 


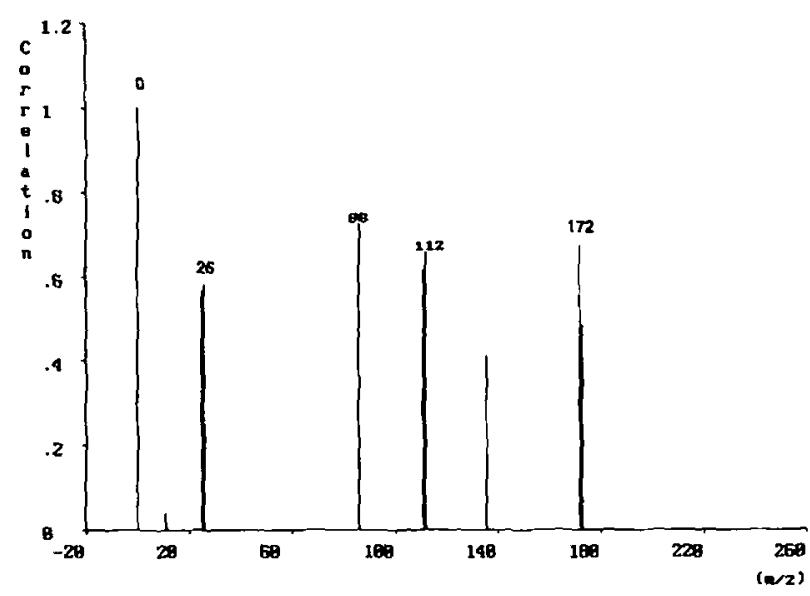

Figure 5. The possible monomer masses for the spectrum in Figure 4.

of rounding of mass-to-charge values due to isotopic contributions and mass defects, and most other possibilities are combinations of these values. The $m / z 25$ and 26 are the difference of the monomer masses, and the 172 is the result of translation of the spectrum by two vinyl acetate units. These values of 86 and $111 \mathrm{u}$ are then entered for solution of the end group mass with the result shown in Figure 6. There are several possible end groups values, which means that several different end groups are present in this polymer; some are chemically unreasonable, such as $m / z 11$ and 22 , which could be basic end groups that, with the addition of a monomer unit of 86 or $111 \mathrm{u}$, would then have chemically reasonable masses of $97,108,122$, and $133 \mathrm{u}$, respectively. From the spectrum of Figure 4, one then can conclude that the sum of the masses of the end groups plus any adduction of $\mathrm{H}^{+}$or $\mathrm{Na}^{+}$is most likely $36,47,61,86,97$, or 111 u. Additionally since $100 \%$ of the abundance cannot be accounted for by one of these masses plus its associated isotopic masses, then there must be more than one end group present.

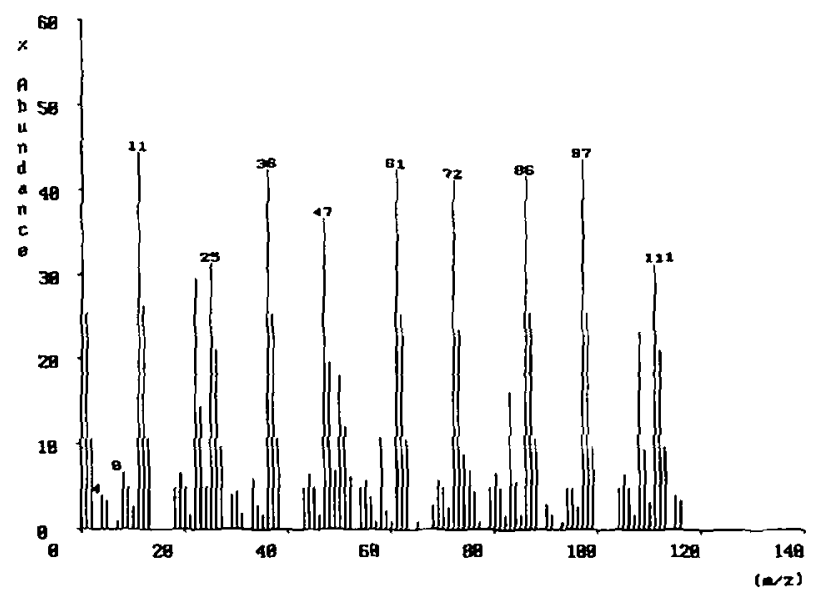

Figure 6. The possible end group masses and the percent of the spectrum in Figure 4 for which they account.

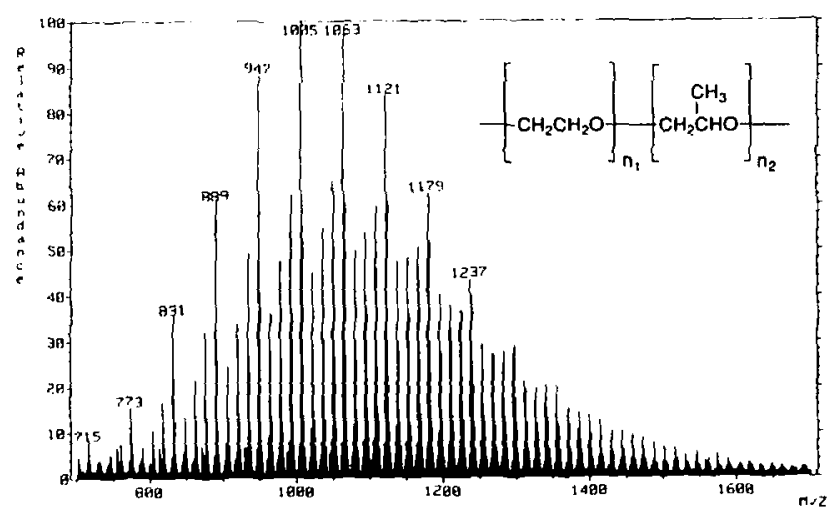

Figure 7. FD mass spectrum of poly(ethylene oxide/propylene oxide).

The FD mass spectrum of poly(ethylene oxide/propylene oxide) molecular weight $1100 \mathrm{u}$ is shown in Figure 7. The distribution extends from below $m /=$ 700 to above 1700 with a maximum around $m / z 1100$. It is readily apparent from the spectrum that a similar pattern of peaks repeats every $m / z 58$. It thus may be possible to manually determine the monomer masses involved in this polymer. However, one would have to enlist the trial and error method to pick possible masses and see if they fit. By application of the MSCOPOL program these data are reduced rapidly to the output in Figure 8 which show the possible monomer masses. The $m / z 58$ is the most likely possibility as well as twice this value and negative values of $m / z-58$ and -116 . Additionally, $m / z 44$ is apparent with its associated combinations with $m / z 58$, for example, $m /=$ -14 is due to $m / z 58$ minus 44 . An additional result shown here that is not obvious from the mass spectrum is that although the propylene oxide, mass $58 \mathrm{u}$, is symmetrically distributed with both positive and negative values, the ethylene oxide, mass $44 u$, shows only positive values. This is interpreted as being due to the method in which the polymer was prepared and the resultant structure. Thus, in the sample there is

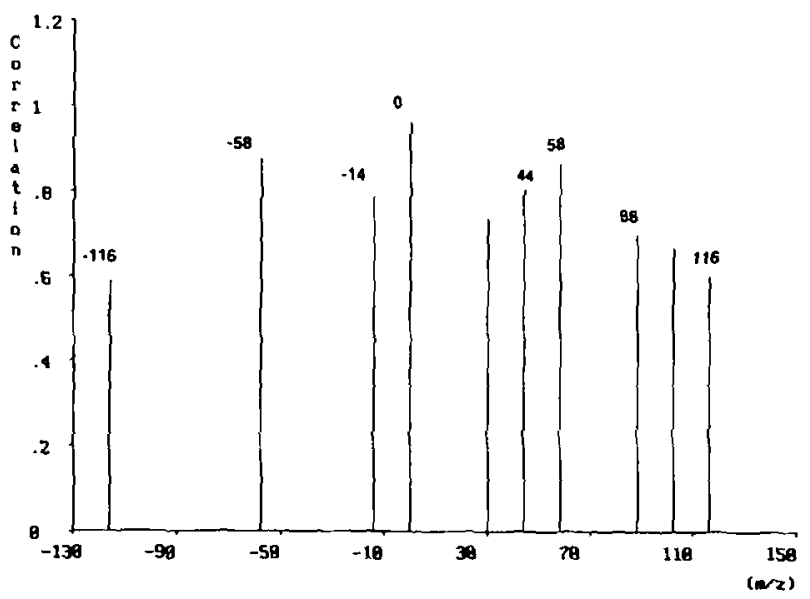

Figure 8. The possible monomer masses for the spectrum in Figure 7. 


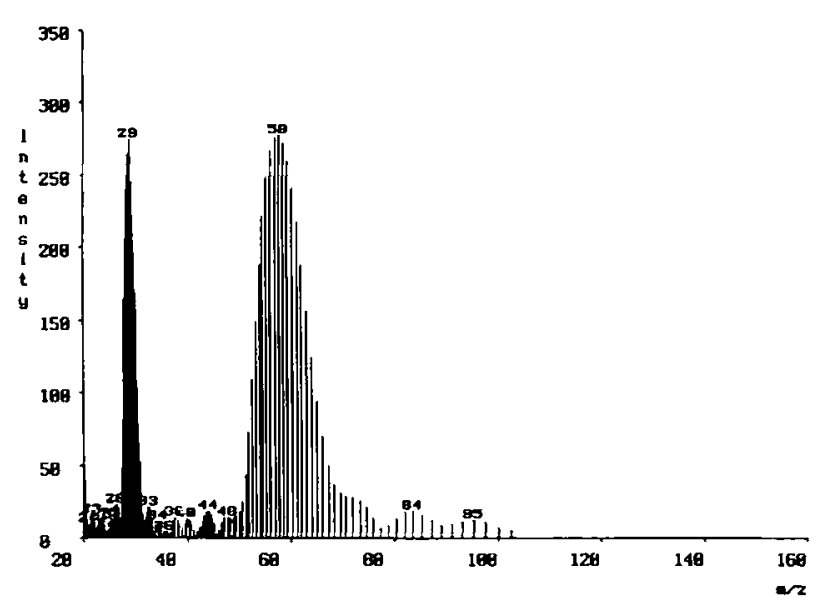

Figure 9. The FFT of the data in Figure 7 shown as the modulus of each Fourier transform datum point versus the reciprocal of its abscissa.

homopolymer of propylene oxide, but no homopolymer of ethylene oxide, and furthermore the copolymer was prepared by addition of the ethylene oxide onto a prepolymer of propylene oxide that resulted in an $A B$ block copolymer.

By application of the Fourier transform method to the spectrum in Figure 7, the result in Figure 9 is obtained. As was expected the periodicity of the monomer mass $58 \mathrm{u}$ is apparent. However, there are several other aspects that render this result of less utility than that obtained previously with correlation methods. Like every FFT algorithm, it is limited by aliasing that results from truncation (finite mass range) and by the finite number of transform points [18]. Moreover, the mass distribution of the polymer is always somewhat bell-shaped instead of rectangular. Consequently, the Fourier transform of the spectrum looks like a series of waves of various amplitudes and widths that peak at masses $M_{i}$ and $M_{i} / 2$, which makes assignment difficult. Additionally the other monomer of mass $44 \mathrm{u}$ is barely apparent as a weak peak. Although the Fourier transform method here gives a less useful result than the correlation method, not enough data are currently available to conclude that this is a general trend.

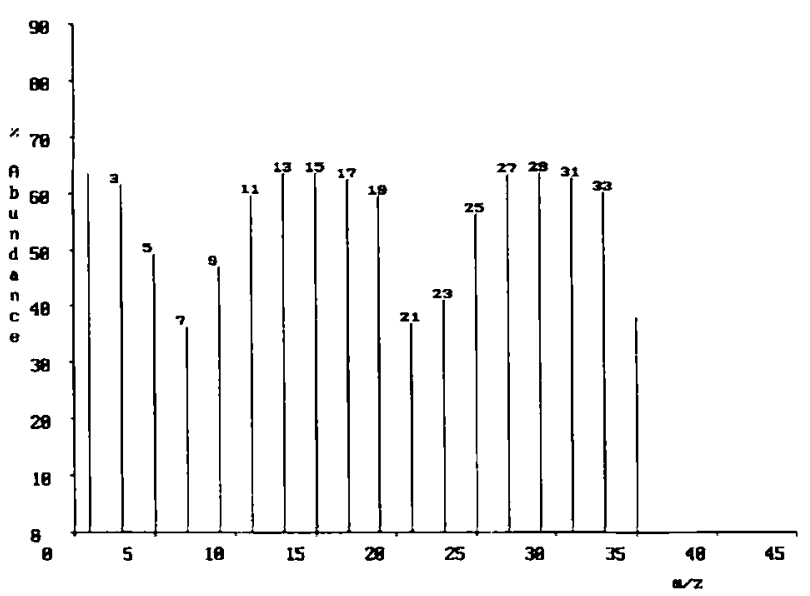

Figure 10. The possible end group masses and the percent of the spectrum in Figure 7 for which they account.

By input of the monomer masses of 44 and $58 \mathrm{u}$ previously determined, the possible end group masses are calculated and shown in Figure 10. As opposed to the polystyrene case, the mass of the end groups is not obvious. This shows the limit of the mathematical treatment in that coincidences can dominate the result. From this one can only conclude that the possible end groups could be one of at least eight values that can form chemically reasonable moieties. Also the addition of one or more monomer masses to the mass of one of these end groups values could make for even more possibilities.

Rather than try to postulate particular end groups by using reasonable polymer chemistry, another approach was taken to mathematically reduce this result. Because the possible values of end groups are known from Figure 8, a reconstruction of the polymer distribution with different values of end groups should show the degree of polymerization required for each mass-to-charge ratio value in the spectrum. This shares some similarities with the method of Montaudo et al. [15] to statistically predict spectra and match them to the experimental result. In Table 1 are shown the various degrees of polymerization required to satisfy the mass spectrum for a variety of end group masses between 15 and $33 \mathrm{u}$. There are two factors based on

Table 1. Copolymer composition computation for monomers of ethylene oxide and propylene oxide"

\begin{tabular}{|c|c|c|c|c|c|c|c|}
\hline \multirow{2}{*}{$\begin{array}{c}\text { End group } \\
\text { mass }\end{array}$} & \multirow{2}{*}{$\begin{array}{c}\% \text { of EO, } \\
\text { mean }\end{array}$} & \multicolumn{3}{|c|}{$n_{1}$} & \multicolumn{3}{|c|}{$n_{2}$} \\
\hline & & Mean & Min. & Max. & Mean & Min. & Max \\
\hline 15 & 49.4 & 9.5 & 8 & 11 & 10.0 & 5 & 15 \\
\hline 17 & 30.1 & 5.5 & 4 & 7 & 13.0 & 8 & 18 \\
\hline 19 & 8.4 & 1.5 & 0 & 3 & 16.0 & 11 & 21 \\
\hline 25 & 84.5 & 18.5 & 17 & 20 & 4.0 & 0 & 8 \\
\hline 27 & 71.8 & 14.5 & 13 & 16 & 6.0 & 1 & 11 \\
\hline 29 & 54.7 & 10.5 & 9 & 12 & 9.0 & 4 & 14 \\
\hline 31 & 35.6 & 6.5 & 5 & 8 & 12.0 & 7 & 17 \\
\hline 33 & 14.3 & 2.5 & 1 & 4 & 15.0 & 10 & 20 \\
\hline
\end{tabular}

${ }^{a} n_{1}$ and $n_{2}$ are the number of monomer units of ethylene oxide (EO) and propylene oxide, respectively 
polymer chemistry that help to reduce the possibilities. First, the percent of ethylene oxide, $M_{1}$, varies from 8.4 to $84.5 \%$ dependent on the end group mass used to reconstruct the polymer distribution. Because the supplier states that the ethylene oxide/propylene oxide monomer ratio in the polymerization is $0.15: 1$, the percent of ethylene oxide in the polymer is most likely approximately $10-15 \%$. This value of monomer ratio also is determined readily by techniques such as pyrolysis gas chromatography [21] and NMR and infrared spectroscopies [13]. This result indicates that 19 and 33 $\mathrm{u}$ are likely masses for end groups. Second, the distribution of $n_{1}$ is narrow in all cases, but the likelihood of producing a distribution of only four monomer units is far greater for low values of $n_{1}$ than for higher values, which leads to further evidence that masses 19 and 33 $\mathrm{u}$ are the most likely values for the end groups of this polymer. The $19 \mathrm{u}$ can be accounted for by termination of the polymer by $\mathrm{H}$ on one end, $\mathrm{OH}$ on the other, and ionization by proton attachment. The $33 \mathrm{u}$ could occur when a methoxy is present instead of a hydroxy group or could simply be the substitution of one propylene oxide for an ethylene oxide in the polymer. This question could then be resolved with a technique such as nuclear magnetic resonance, which would show if there was an oxygen- or carbon-bound methyl group in the polymer.

\section{Conclusion}

We have developed a program to aid in the interpretation of copolymer mass spectra. There is a wealth of information available in these spectra and it is usually difficult for the analyst to extract all the useful information. The program determines the most likely masses of the monomers and from these values then determines the most likely masses for the end groups. By calculating the monomer ratio and degree of polymerization as a function of end group mass, the end group result can be further simplified.

The possibilities can be reduced greatly by then considering chemical information such as logical compositions and possible end groups from known initiators and chain transfer agents. Often a single unambiguous result is not obtained. However, the greatest asset of this approach is that all possibilities are examined in contrast to the manual approach in which the analyst is often satisfied when a fit is found, and further inquiry is halted.

Future work includes expansion of the data bases to include most reasonably common monomers, initia- tors, and chain transfer agents and their product end groups. This information will be contained in the output. Further refinement of the isotopic contribution correction is also underway. Finally the method will be extended to polymers that contain more than two monomers.

\section{Acknowledgments}

The authors are grateful to D. E. Karr for helpful discussions and thank Rohm and Haas Company for support of this work.

\section{References}

1. Schulten, H. R.; Lattimer, R. P. Mass Spectrom. Rẽ. 1984, 3, 231.

2. Saito, J.; Waki, H.; Teramae, N.; Tanaka, S. Progr. Org. Coat. $1988,75,311$.

3. Rollins, K.; Scrivens, J. H.; Taylor, M. J.; Major, H. Rapid Comman. Mass Spectrom. 1990, 4, 355.

4. Montaudo, G. Raprid Commmm. Mass Spectrom. 1991, 5, 95.

5. Ballistreri, A.; Garozzo, D.; Ciuffrida, M.; Montaudo, G.; Filippi, A.; Guaita, C.; Manaresi, P.; Pilati, F. Macromolecules 1987, 20, 1029.

6. Nuwaysir, L. M.; Wilkins, C. L.; Simonsick, W. J., Jr. I. Am. Soc. Mass Spectrom. 1990, 1, 66.

7. Cotter, R. I.; Honovich, I. P.; Olthotf, I. K.; Lattimer, R. P. Macromolecules 1986, 19, 2996.

8. Bletsos, I. V.; Hercules, D. M.; vanLeyen, D.; Benninghoven, A. Marromolecules 1987, 20, 407.

9. Belu, A. M.; Hunt, M. O., Jr.; DeSimone, J. M.; Linton, R. W. Macromolecule's 1994, 27, 1905.

10. Danis, P. O.; Karr, D. E.; Maver, F.; Holle, A.; Watson, C. H Org. Mass Spectrom. 1992, 27, 843.

11. Bahr, U.; Deppe, A.; Karas, M.; Hillenkamp, F.; Giessmann U. Amal. Che'm. 1992, 64, 2866.

12. Danis. Г. O.; Karr, D. E.; Simonsick, W. J.; Wu, D. T. Macromolicule's 1995, 28, 1229.

13. Koenig, J. L. Spritrosioply of Polymers; Washington, DC: American Chemical Society, 1991.

14. Adamson, K.; Simonsick, W. J., Jr. I. Appl. Polym. Sici: Apll Polym. Sylmy. 1990, 45, 339.

15. Montaudo, M. S.; Ballistreri, A.; Montaudo, G. Mncomblecule's $1991,24,5051$.

16. Georgakopoulos, C. G.; Statheropoulos, M; Kontoyannakos, J.; Parissakis, C. Chimo. Inlell. Lal. Syst. 1993, 19, 75.

17. Mclafferty, F. W.; Stauffer, D. B. I. Chem. Inf. Comput. Sit. $1985,25,245$, and references therein.

18. Brigham, E. O. The Fast Fourier Transform and its Applications; Englewood Clifts, NJ: Prentice-Hall, 1988.

19. Brandrup, J.; Immergut, E. H., Eds. Polymer Handlook, 3rd ed.; New York: Wilev, 1989

20. Stevens, M. P. Polymor Ch'mistry, An Introduction, 2nd ed.; New York: Oxford University Press, 1990.

21. Liebman, S. A.; Levy. E. J. Pyrolysis and CC in Polymel Amalysis; New York: Marcel Dekker, 1484. 